\title{
Extraventricular Neurocytoma in Parietal Lobe
}

\author{
Katherine Gallego-Henao ${ }^{1}$ José M. Ramos-Delgado1, $\odot$ Angelica Moreno-Blanco² \\ Aureliano Placido-Méndez ${ }^{2}$ Antonio Zarate-Mendez ${ }^{1}$
}

1Department of Neurosurgery, Centro Médico Nacional "20 de Noviembre" Instituto de Seguridad y Servicios Sociales de los Trabajadores del Estado (ISSSTE), Mexico City, México

${ }^{2}$ Department of Pathology, Centro Medico Nacional "20 de Noviembre" Instituto de Seguridad y Servicios Sociales de los Trabajadores del Estado (ISSSTE), Mexico City, México

\begin{abstract}
Address for correspondence José M. Ramos-Delgado, MD, Department of Neurosurgery, Centro Médico Nacional "20 de Noviembre" Instituto de Seguridad y Servicios Sociales de los Trabajadores del Estado (ISSSTE), Mexico City, México (e-mail: jmiguelrd1@gmail.com).
\end{abstract}

Indian J Neurosurg 2021;10:144-147.

\begin{abstract}
Keywords

- EVN

- extraventricular neurocytoma

- central neurocytoma

Extraventricular neurocytoma (EVN) was classified as a World Health Organization (WHO) grade II tumor; however, EVN is not fully understood; it presents a variable histological feature that included oligodendroglioma-like, neuropil-like matrix, ganglion or gangloid cells, perivascular pseudorosettes, vessel hyalinization, calcifications, and myxoid degeneration. In some very rare cases, atypical histological features such as increased mitotic figures, focal necrosis, endothelial cell proliferation, and $\mathrm{K}_{\mathrm{i}}-67$ index of $>2 \%$ made this tumor more aggressive and more susceptible to recur. We present the case of a young patient who presents with a 2-year history of seizure without other symptoms. Magnetic resonance imaging reveals a parietal lobe and well-circumscribed lesion treated by gross total resection and adjuvant radiotherapy. Clear guidelines to treat this kind of lesions are not well established and there is not a consensus of correct treatment in these tumors.
\end{abstract}

\section{Case Presentation}

A 35-year-old male patient, with no chronic-degenerative history, presents with vertigo and headache which began 9 months before its assessment, and later a generalized tonic-clonic seizure episode, which remitted with anticonvulsants. A neuroimaging study was performed, evidencing left parietal tumor involving angular and supramarginal gyrus (-Fig. 1). Neurological examination was unremarkable.

Gross total resection was performed obtaining hemorrhagic liquid of $\sim 20 \mathrm{cc}$ and lesion of cystic aspect; the entire capsule was removed without complications. After a surgical event, the patient maintains without neurological deficit.

Our pathology department reports hematoxylin and eosin stains that show brain parenchyma with the presence of a tumoral lesion of rounded cells, with clear cytoplasm and hyperchromatic nuclei, embedded in a fibrillar matrix (-Fig. 2).

Given these findings, immunostaining is performed that reports positivity in tumor cells for synaptophysin ( - Fig. 3 ) and negativity for glial fibrillar acid protein ( - Fig.4), epithelial membrane antigen ( - Fig. 5), and progesterone receptors ( - Fig. $\mathbf{6}$ ).

Considering these results in the immunohistochemical staining, the lesion is reported as a World Health Organization (WHO) grade II extraventricular neurocytoma (EVN).

\section{Discussion}

Although central neurocytoma was first described in 1982 by Hassoun et $\mathrm{al}^{1}$, the first case of a lesion with similar characteristics of central neurocytoma outside the ventricular system was described in 1989 by Ferreol et $\mathrm{al}^{2}$ in a 65-year-old male treated only with gross total resection. published online April 20, 2021
DOI https://doi.org/

$10.1055 / \mathrm{s}-0041-1727546$

ISSN 2277-954X
(C) 2021. Neurological Surgeons' Society of India.

This is an open access article published by Thieme under the terms of the Creative Commons Attribution-NonDerivative-NonCommercial-License, permitting copying and reproduction so long as the original work is given appropriate credit. Contents may not be used for commercial purposes, or adapted, remixed, transformed or built upon. (https://creativecommons.org/licenses/by-nc-nd/4.0/).

Thieme Medical and Scientific Publishers Pvt. Ltd. A-12, 2nd Floor, Sector 2, Noida-201301 UP, India 

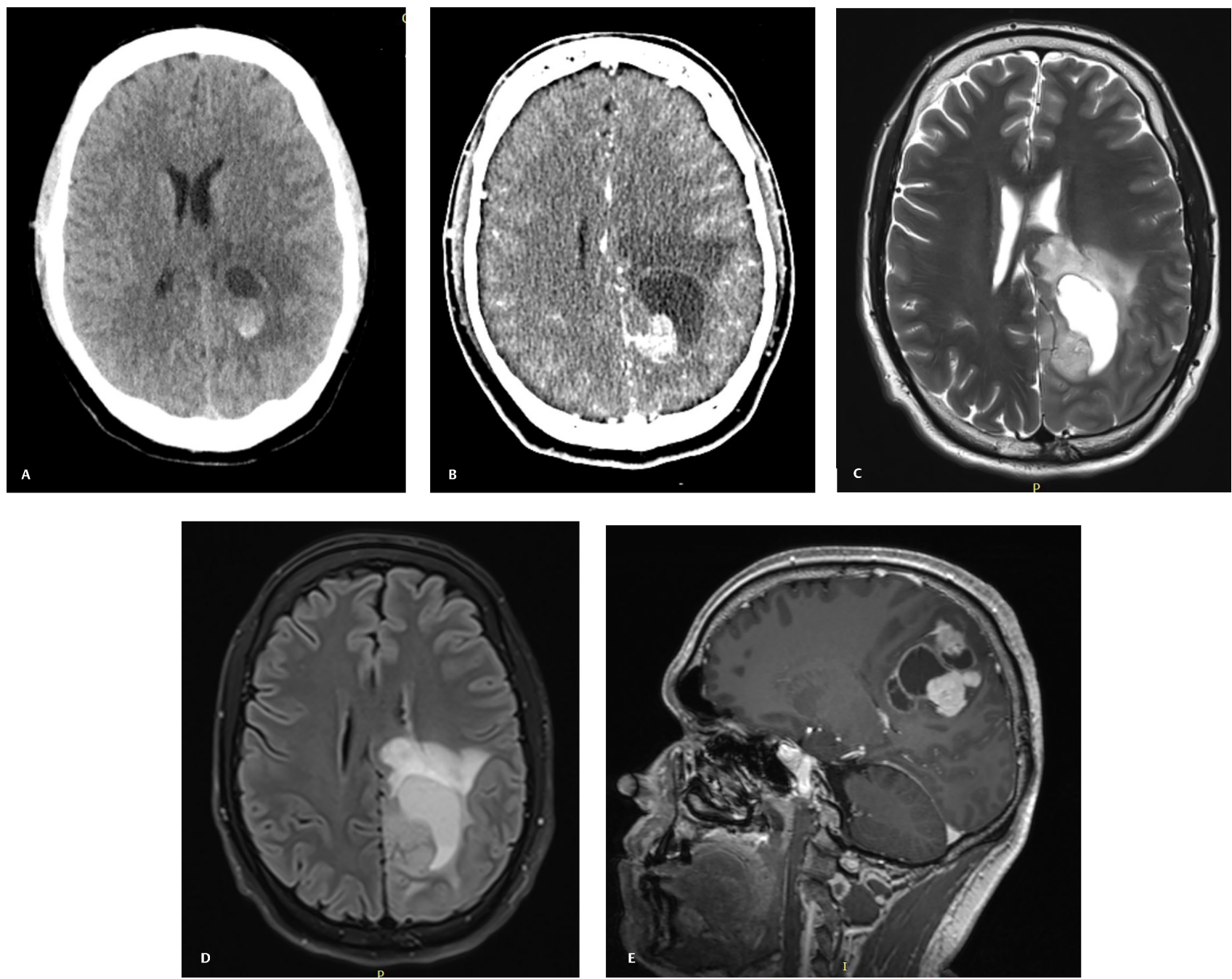

Fig. 1 (A) Computed tomography (CT) showing parietal periventricular lesion, heterogenous with hemorrhagic aspect. (B) Contrasted CT showing mild enhancement of the solid portion. (C) Magnetic resonance imaging showing T2 well-circumscribed lesion of cystic aspect with peripheric solid portion. (D) Fluid-attenuated inversion recovery demonstrating mild edema. (E) Sagittal projection with gadolinium demonstrating homogenous enhancement of the solid portion and capsular ring enhancement.

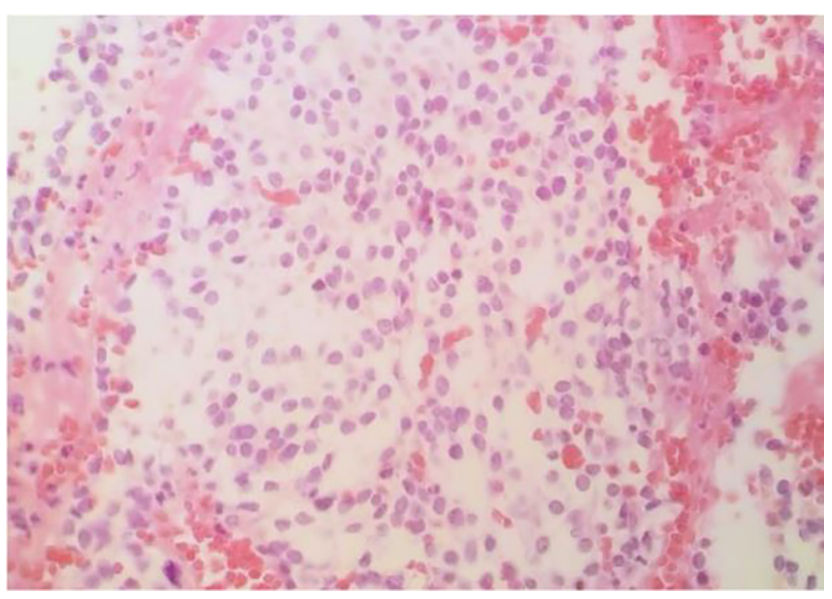

Fig. 2 Hematoxylin and eosin, 40x. Rounded cells, clear cytoplasm, and hyperchromatic nuclei, embedded in a fibrillar matrix.

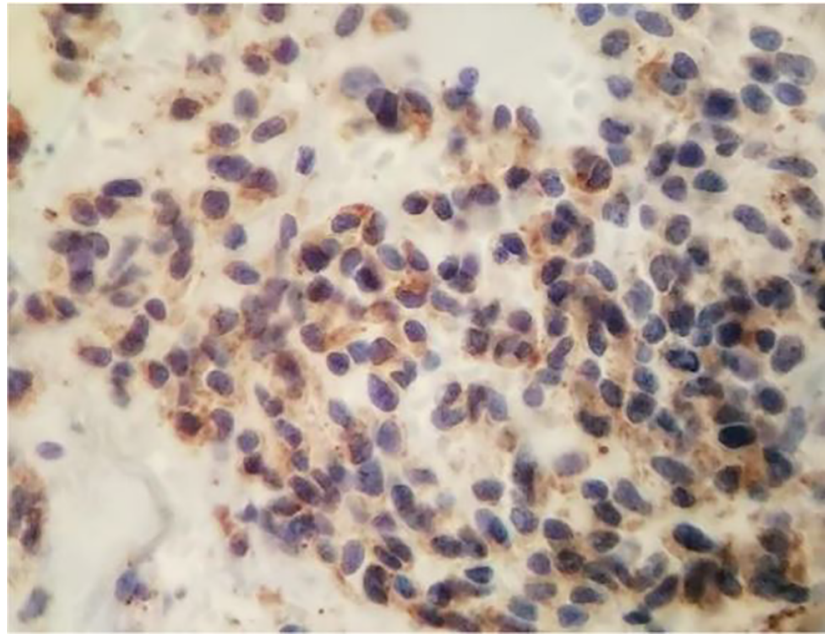

Fig. 3 Immunohistochemistry, 40x. Synaptophysin positive. 


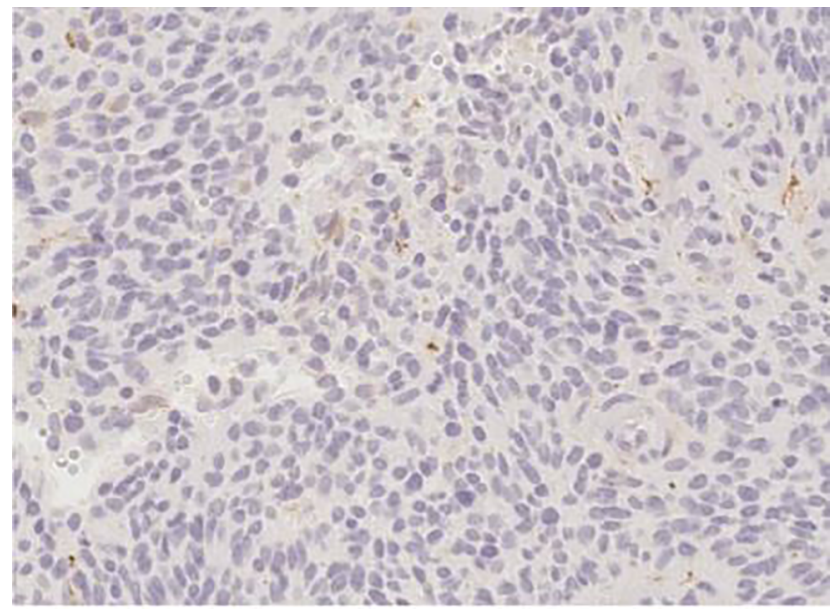

Fig. 4 Immunohistochemistry, 20x. Glial fibrillar acid protein negative.

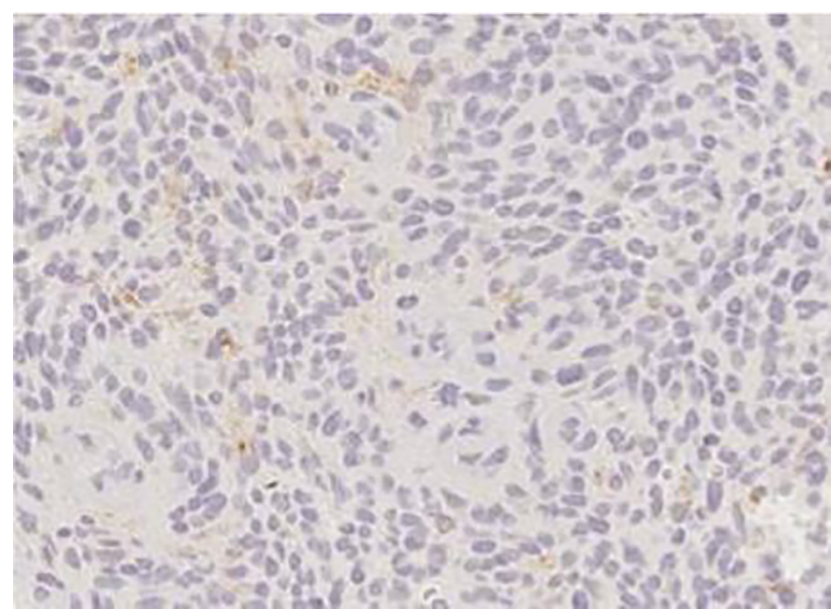

Fig. 5 Immunohistochemistry, 20x. Epithelial membrane antigen negative.

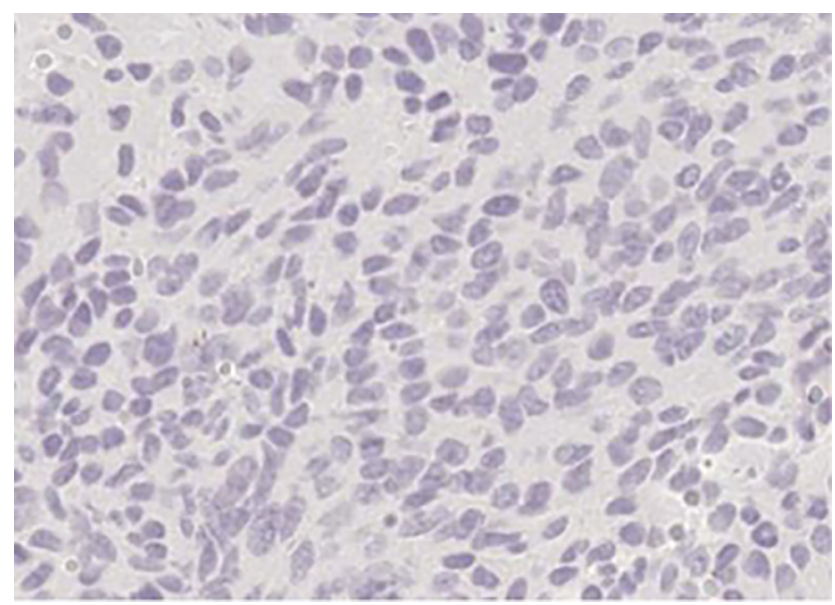

Fig. 6 Immunohistochemistry, 40x. Progesterone receptors negative.

EVN has histopathological, immunohistochemical, and ultrastructural similarities to central neurocytoma but outside of the ventricular system and in some cases with different behavior. The WHO in 2007 classified them as a separate entity and in the review of 2016, it was classified as a Grade II. ${ }^{3}$

EVNs are a rare entity associated with a more aggressive biological behavior than central neurocytoma; the largest epidemiology study for these tumors reports an incidence rate of 0.009 per 100,000 for EVN with rates per location in frontal lobe 0.002 , temporal lobe 0.001 , parietal lobe 0.001 , ventricle not otherwise specified 0.022 , and cerebellum not otherwise specified $0.002 .{ }^{4}$ In the case of our patient, the lesion was evidenced at the parietal level, being the third most frequent location for this type of pathology. Many reports a mean age of presentation for EVNs around 27 years, the range varies from 2 to 75 years, and pediatric presentation as rare but known; however, the peak incidence rate was noted in the 20 to 34 age group. 4,5

Symptom onset may vary from 3 months to 10 years and the average duration of clinical symptoms and signs of 32.4 months. ${ }^{5}$ The most common clinical presentation is related to a raise intracranial pressure but may vary depending on tumor location, from seizure to gait disturbances among others such as numbness or motor alterations. Increase in intracranial pressure symptoms was a common observation in many articles; however, in some cases it was disproportionate to the size of the lesion, especially in atypical lesions.

On computed tomography (CT) and magnetic resonance imaging (MRI), EVNs appear as solid or cystic well-circumscribed lesions showing heterogeneous contrast enhancement, as shown in our case. EVNs should be included in the differential diagnosis when a large parenchymal mass with cystic necrosis, calcification, hemorrhage, and extensive enhancement. ${ }^{6}$ Also, Liu k et al report new findings concerned focal infiltration and complex internal architecture, isointensity of the solid part on T1-weighted imaging, and nonenhancement of some tumors. ${ }^{7}$

The solid portion may have a variable appearance on CT and MRI, depending on the cellularity and degree of calcification. They are frequently calcified (10\%) and may or may not be associated with peritumoral edema. Hemorrhage has been reported sporadically. EVNs with gangloid differentiation tended to have more of a cystic component and heterogeneous enhancement appearance. ${ }^{7}$

Definitive diagnosis is made on histopathological examination, and frequently microscopic appearance mimics that of oligodendrocytomas. As shown in our case, these lesions are composed of uniform round/oval cells, which had round or oval nuclei with only speckled chromatin and an occasional nucleolus. Immunohistochemical staining of EVN shows diffuse and strong positivity for synaptophysin as shown in - Fig. $\mathbf{3}$, and in some cases it has immunoreactivity for neuron-specific nuclear protein. Mitoses are rare or absent, and there was mild endothelial cell proliferation; however, in atypical cases, tumor cells with hyperchromatic nuclei are closely packed, focal necrosis, and prominent endothelial cell proliferation. Atypical cases have more than four mitoses per 50 high-power fields and a $\mathrm{K}_{\mathrm{i}}-67$ proliferation index $>2 \% .{ }^{8-10}$ 
Optimal treatment of EVN is not determined yet, however, surgical removal is the treatment and maximal safe resection should be the goal. Some authors report malignant behaviors of EVN, including recurrence. ${ }^{11}$ The definitive efficacy of postoperative adjuvant radiotherapy and chemotherapy is not clear, but the recommended radiation dosage is 54 to $60 \mathrm{~Gy} .{ }^{5}$ In our case, the dose adjusted for treatment was $54 \mathrm{~Gy}$ divided into 30 cycles.

\section{Conclusion}

EVNs are considered infrequent tumors of the central nervous system with a similar appearance to oligodendrogliomas; it is important to differentiate them from other lesions due to their imaging and immunohistochemical characteristics. Complete surgical resection is the treatment of choice in these cases, in terms of adjuvant radiotherapy, mainly for local control of the disease, and is supported by the literature for cases where complete macroscopic resection was not achieved. Finally, it should be noted that in atypical neurocytomas with rapid local recurrence or radiation failure, concomitant use of chemotherapy is recommended; however, there are no well-established treatment guidelines due to the infrequency of these tumors.

\section{Conflict of Interest}

None declared.

\section{References}

1 Hassoun J, Gambarelli D, Grisoli F, et al. Central neurocytoma. An electron-microscopic study of two cases. Acta Neuropathol 1982;56(2):151-156
2 Ferreol E, Sawaya R, de Courten-Myers GM. Primary cerebral neuroblastoma (neurocytoma) in adults. J Neurooncol 1989;7(2):121-128

3 International Agency for Research on Cancer, (;2016) WHO Classification of Tumors of the Central Nervous System (IARC WHO Classification of Tumours). 4th Revised edition. Lyon: International Agency for Research on Cancer

4 Tish S, Habboub G, Jones J, et al. The epidemiology of central and extraventricular neurocytoma in the United States between 2006 and 2014. J Neurooncol 2019;143(1):123-127

5 Patil AS, Menon G, Easwer HV, Nair S. Extraventricular neurocytoma, a comprehensive review. Acta Neurochir (Wien) 2014;156(2):349-354

6 Yang GF, Wu SY, Zhang LJ, Lu GM, Tian W, Shah K. Imaging findings of extraventricular neurocytoma: report of 3 cases and review of the literature. AJNR Am J Neuroradiol 2009;30(3): 581-585

7 Liu K, Wen G, Lv XF, et al. MR imaging of cerebral extraventricular neurocytoma: a report of 9 cases. AJNR Am J Neuroradiol 2013;34(3):541-546

8 Chen F, Jin R, Wu X, Dong Z, Chen D. Extraventricular neurocytoma in the left frontal lobe: a case report and literature review. World Neurosurg 2018;112:178-181

9 Xu L, Ouyang Z, Wang J, et al. A clinicopathologic study of extraventricular neurocytoma. J Neurooncol 2017;132(1): 75-82

10 Brat DJ, Scheithauer BW, Eberhart CG, Burger PC. Extraventricular neurocytomas: pathologic features and clinical outcome. Am J Surg Pathol 2001;25(10):1252-1260

11 Moriguchi S, Yamashita A, Marutsuka K, et al. Atypical extraventricular neurocytoma. Pathol Int 2006;56(1):25-29 